\title{
Methods for Determination of Mean Speckle Size in Simulated Speckle Pattern
}

\author{
I. Hamarová ${ }^{1}$, P. Šmíd ${ }^{1}$, P. Horváth ${ }^{2}$, M. Hrabovský ${ }^{2}$ \\ ${ }^{1}$ Institute of Physics of the Academy of Sciences of the Czech Republic, Joint Laboratory of Optics of Palacky University \\ and Institute of Physics of the Academy of Sciences of the Czech Republic, 17. listopadu, 12, 77141, Olomouc, Czech \\ Republic, ivana.hamarova@upol.cz,petr.smid@upol.cz \\ ${ }^{2}$ Regional Centre of Advanced Technologies and Materials, Joint Laboratory of Optics of Palacky University and Institute \\ of Physics of the Academy of Sciences of the Czech Republic, Faculty of Science, Palacký University, 17. listopadu, 12, \\ 77141, Olomouc, Czech Republic, pavel.horvath@upol.cz, miroslav.hrabovsky@upol.cz
}

This paper deals with computation of mean speckle size in a speckle pattern generated through a numerical simulation of speckle after reflection of a Gaussian beam off a rough object's surface. Within this simulation various speckle patterns are obtained by means of change in a parameter of the Gaussian beam. The mean speckle size is computed through two approaches using both the two-dimensional and the one-dimensional normalized autocorrelation function in intensity. Additionally, we propose a distinct optimization of the determination of the mean speckle size by reduction of intensity values representing detected speckle patterns. Results of the determination of the mean speckle size are compared with theoretical predictions.

Keywords: Simulation, speckle, speckle pattern, mean speckle size, autocorrelation function.

\section{INTRODUCTION}

$\mathrm{A}$ N OPTICAL speckle effect [1] arises when a coherent optical beam is either reflected from a rough surface or propagates through a medium having scattering centers randomly distributed. In a detection plane we can observe a speckle pattern consisting of dark and bright speckles. This pattern results from the interference of multiple coherent spherical waves, emitted by point sources forming the object's surface in the case of reflection or the medium in the case of transmission [1].

In general, a speckle parameter such as mean speckle size depends both on properties of the light and properties of the random surface or medium. However, for perfectly coherent light the dependence on the random scatterer is almost negligible if the scatterer introduces path differences greater than one wavelength [1]. The mean speckle size, as a very important parameter of the speckle pattern, is of great importance to practical applications, e.g., measurement of the roughness of surfaces [2], detection of the scattering center concentration in a biological fluid [3], the particle aggregation [4] or determination of the optical thickness and the particle size in the scattering media [5]. Nevertheless, this paper is focused on determining the mean speckle size influenced purely by the properties of the light beam.

The mean speckle size of a speckle pattern corresponds to the width of a normalized autocorrelation function $r_{I}$ of intensity $I$ observed in a detection plane $\left(x^{\prime}, y^{\prime}\right)$ [1], [4], [5]. This paper deals with the calculation of the normalized autocorrelation function $r_{I}$ and the subsequent estimation of the mean speckle sizes $\alpha_{x^{\prime}}$ and $\alpha_{y^{\prime}}$ in both $x^{\prime}$ - and $y^{\prime}$-axis directions, while two approaches of computation are applied and compared to each other.

Within the first approach, which is more conventional than the second one, the mean speckle size is defined as a value where the vertical (horizontal) profile of the twodimensional (2D) normalized autocorrelation function $r_{I}$ of intensity $I$ decreases to $1 / 2$ [4]. However, substituting the value $1 / \mathrm{e}$ for $1 / 2$ is proposed in the presented paper.

The second approach of the mean speckle size estimation based on an alternative algorithm is proposed in [3]. Each vertical (horizontal) intensity profile of the speckle pattern is extracted as a series of values. For each profile the onedimensional (1D) normalized autocorrelation function of intensity $I$ is computed. The mean speckle size for the $i$-th profile (the profile speckle size) is defined as a value where the 1D normalized autocorrelation function $r_{I}$ decreases to $1 / \mathrm{e}$. The mean speckle size for the whole detected area is defined as an average of the profile speckle size. The advantage of the alternative algorithm is that it is faster than the conventional algorithm based on the computation of the 2D autocorrelation function of the 2D intensity signal [3].

Within the approach based on the computation of the $1 \mathrm{D}$ autocorrelation function $r_{I}$ of intensity $I$, we propose optimization of the determination of the mean speckle size by reducing intensity values representing the detected speckle pattern. Hence, the main advantage of the optimized method rests not only on less computer time consuming algorithm, but also on the possibility of determining the mean speckle size from a smaller amount of the detected intensity values.

This paper uses the simulation model (Fig.1.) according to [6-8] and the way of reducing intensity values (decimation) presented in [6]. The aim of the paper [6] is to present an extension of the one-dimensional speckle correlation method, which is primarily intended for determination of one-dimensional object's translation, for detection of general in-plane object's translation. The results presented in [6] show that the use of decimation enables the proposed 1D cross-correlation method to successfully replace the time-consuming 2D cross-correlation method. Unlike [6], this paper presents that the decimation can also be useful in evaluation of the mean speckle size and it provides 
reasonable results. However, 1D autocorrelation function needs to be computed in several rows and columns (not in all rows as in [3]) while in [6] 1D cross-correlation function is computed only once for each axis direction from 1D signal acquired after numerical processing of $2 \mathrm{D}$ speckle pattern.

For simulation of the speckle effect the numerical simulation model [7], [8] is used. The main part of the program code is comprised of the Fresnel-Kirchhoff diffraction integral calculation through the rectangle method. It computes the distribution of the complex amplitude of speckle field at a distance from the object's surface. The object's surface is represented by a matrix of values defining random variable surface roughness. The presented numerical model enables to select initial parameters of simulation independently, including nonzero angle of observation [8].

Within the presented numerical model the object generating the speckle effect is illuminated by a Gaussian beam propagating from its waist situated at a distance $L_{s}$ from the object. A beam radius $\omega$ in the object plane $(x, y)$ varies in consequence of controlled variation of a beam radius $\omega_{o}$ at the beam's waist. Then several speckle patterns with different speckle sizes are simulated, since the mean speckle size depends on the size of the beam spot in the object plane [1]. Numerical results of the determination of the mean speckle size for six different simulated speckle patterns for the Gaussian beam radii $\omega_{o}=40 \mu \mathrm{m}, 50 \mu \mathrm{m}$, $60 \mu \mathrm{m}, 70 \mu \mathrm{m}, 80 \mu \mathrm{m}$ and $90 \mu \mathrm{m}$ are compared with the results obtained from theoretical relations.

\section{SUBJECT \& METHODS}

As mentioned above, the mean speckle size is defined as width of the normalized autocorrelation function $r_{I}$ of intensity $I$ of the speckle pattern observed in the detection plane $\left(x^{\prime}, y^{\prime}\right)$. For the 2D normalized autocorrelation function $r_{I}$ of intensity $I$ one can write [1]-[3]

$$
r_{I}\left(\Delta x^{\prime}, \Delta y^{\prime}\right)=\frac{\left\langle I\left(x^{\prime}, y^{\prime}\right) I\left(x^{\prime}-\Delta x^{\prime}, y^{\prime}-\Delta y^{\prime}\right)\right\rangle-\left\langle I\left(x^{\prime}, y^{\prime}\right)\right\rangle^{2}}{\left\langle I\left(x^{\prime}, y^{\prime}\right)^{2}\right\rangle-\left\langle I\left(x^{\prime}, y^{\prime}\right)\right\rangle^{2}}
$$

Let us denote by $\alpha_{x}{ }^{2 \mathrm{D}}$ and $\alpha_{y^{\prime}}{ }^{2 \mathrm{D}}$ the mean speckle sizes (radii) defined as values where both the horizontal and the vertical profiles of the function (1) decrease to $1 / \mathrm{e}$, i.e. $r_{I}\left(\alpha_{x}{ }^{2 \mathrm{D}}, 0\right)=1 / \mathrm{e}$ and $r_{I}\left(0, \alpha_{y}{ }^{2}{ }^{2 \mathrm{D}}\right)=1 / \mathrm{e}$. Let us denote this method by the method of 2D correlation.

By analogy with (1), the 1D normalized autocorrelation functions $r_{I}$ of intensity $I$ from a selected row and column of the matrix detector can be written as

$$
\begin{aligned}
& r_{I}\left(\Delta x^{\prime}\right)=\frac{\left\langle I\left(x^{\prime}\right) I\left(x^{\prime}-\Delta x^{\prime}\right)\right\rangle-\left\langle I\left(x^{\prime}\right)\right\rangle^{2}}{\left\langle I\left(x^{\prime}\right)^{2}\right\rangle-\left\langle I\left(x^{\prime}\right)\right\rangle^{2}}, \\
& r_{I}\left(\Delta y^{\prime}\right)=\frac{\left\langle I\left(y^{\prime}\right) I\left(y^{\prime}-\Delta y^{\prime}\right)\right\rangle-\left\langle I\left(y^{\prime}\right)\right\rangle^{2}}{\left\langle I\left(y^{\prime}\right)^{2}\right\rangle-\left\langle I\left(y^{\prime}\right)\right\rangle^{2}} .
\end{aligned}
$$

Let us denote by $\alpha_{x^{\prime}}{ }^{1 \mathrm{D} i}$ and $\alpha_{y^{\prime}}{ }^{1 \mathrm{D} j}$ the values at which functions (2) and (3) are equaled to 1/e, i.e. $r_{I}\left(\alpha_{x^{\prime}}{ }^{1 \mathrm{D} i}\right)=1 / \mathrm{e}$ and $r_{I}\left(\alpha_{y^{\prime}}{ }^{1 D j}\right)=1 / \mathrm{e}$. The values of $\alpha_{x^{\prime}}{ }^{1 \mathrm{D} i}$ and $\alpha_{y^{\prime}}{ }^{1 \mathrm{D} j}$ define the profile speckle sizes (radii) from the $i$-th row and $j$-th column of a matrix detector. Then averages

$$
\alpha_{x^{\prime}}^{1 \mathrm{D}}=\frac{1}{m} \sum_{i=1}^{m} \alpha_{x^{\prime}}^{1 \mathrm{D} i}
$$

and

$$
\alpha_{y^{\prime}}{ }^{1 \mathrm{D}}=\frac{1}{n} \sum_{j=1}^{n} \alpha_{y^{\prime}}{ }^{1 \mathrm{D} j}
$$

computed for $m$ rows and $n$ columns of the matrix detector $(m \times n)$ define the mean speckle sizes for the whole matrix detector [3]. Let us denote this method by the method of $1 \mathrm{D}$ correlation.

Nevertheless, we can optimize the method of 1D correlation by decimation [9] of the 2D intensity signal representing the speckle pattern, which is based on reduction in resolution of the $2 \mathrm{D}$ intensity signal by skipping of a certain amount of the intensity values within rows and columns of the matrix detector. Let us denote by the factor $\Delta$ the distance between rows (columns) of the matrix detector, which are used within the optimized method of 1D correlation. Then the mean speckle sizes $\alpha_{x^{\prime}}{ }^{1 \mathrm{D}}$ and $\alpha_{y^{\prime}}{ }^{1 \mathrm{D}}$ computed from a decimated $2 \mathrm{D}$ intensity signal can be expressed as

$$
\alpha_{x^{\prime}}{ }^{1 \mathrm{D}}=\frac{1}{m_{s}} \sum_{i=0}^{m_{s}} \alpha_{x^{\prime}}{ }^{1 \mathrm{D}(i \Delta+1)}, m_{s}=\text { Floor }(m / \Delta)-1
$$

and

$$
\alpha_{y^{\prime}}{ }^{1 \mathrm{D}}=\frac{1}{n_{s}} \sum_{j=0}^{n_{s}} \alpha_{y^{\prime}}{ }^{1 \mathrm{D}(j \Delta+1)}, n_{s}=\operatorname{Floor}(n / \Delta)-1
$$

where Floor $(x)$ gives the greatest integer less than or equal to $x$.

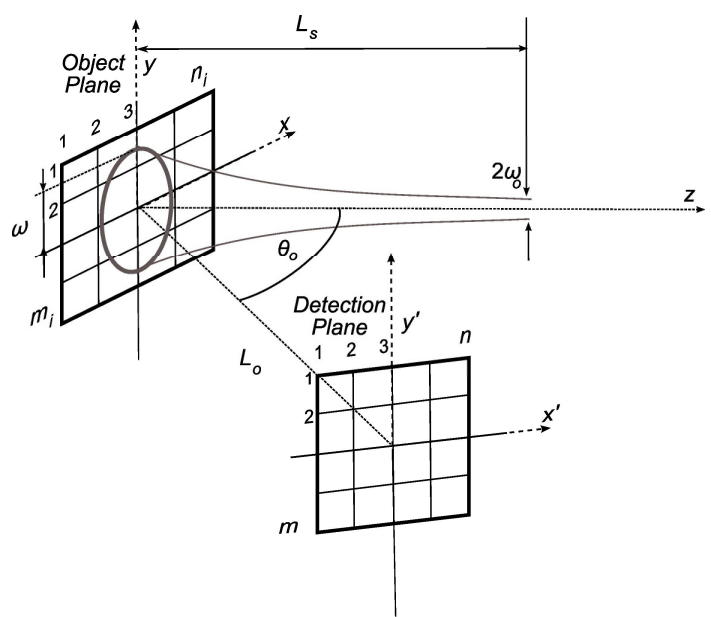

Fig.1. The geometrical arrangement for detection of the speckle pattern used within the numerical simulation. 
In this paper values $\alpha_{x^{\prime}}{ }^{2 \mathrm{D}}$ and $\alpha_{y^{\prime}}{ }^{2 \mathrm{D}}, \alpha_{x^{\prime}}{ }^{1 \mathrm{D}}$ and $\alpha_{y^{\prime}}{ }^{1 \mathrm{D}}$ of the mean speckle sizes in the simulated speckle patterns are determined by means of the above-mentioned methods (the method of 2D correlation and 1D correlation, respectively). Fig.1. shows the geometrical arrangement for detection of the speckle pattern used within the numerical simulation. In addition, stated values are simultaneously compared with theoretical mean speckle sizes $\alpha_{x}$, and $\alpha_{y}$ obtained from theoretical relations derived as follows.

Let us consider that the normalized autocorrelation function $r_{I}(1)$ of intensity $I$ observed in the detection plane $\left(x^{\prime}, y^{\prime}\right)$ can also be expressed by means of intensity distribution $P(x, y)$ of an illuminating beam in the object plane $(x, y)[1],[5]$

$$
r_{I}\left(\Delta x^{\prime}, \Delta y^{\prime}\right)=\left|\frac{\iint_{-\infty}^{\infty} P(x, y) \exp \left[i \frac{2 \pi}{\lambda L_{o}}\left(x x^{\prime}+y y^{\prime}\right)\right] d x d y}{\iint_{-\infty}^{\infty} P(x, y) d x d y}\right|^{2}
$$

where $L_{o}$ is the distance between the object plane $(x, y)$ and the detection plane $\left(x^{\prime}, y^{\prime}\right)$ and $\lambda$ is the wavelength of the light. In the case of illumination by the Gaussian beam the intensity distribution $P(x, y)$ in the object plane $(x, y)$ is [10]

$$
P(x, y)=\left(\frac{\omega_{o}}{\omega}\right)^{2} \exp \left(-2 \frac{x^{2}+y^{2}}{\omega^{2}}\right)
$$

where $\omega_{0}$ and $\omega$ are the radii of the Gaussian beam at its waist and in the object plane $(x, y)$ at a distance $L_{s}$ from the waist, respectively (Fig.1.). The radius $\omega$ is determined as [10]

$$
\omega=\omega_{o} \sqrt{1+\left(\frac{L_{s} \lambda}{\omega_{o}^{2} \pi}\right)^{2}} .
$$

After substituting (9) into (8) the normalized autocorrelation function $r_{I}$ of intensity $I$ can be written as

$$
r_{I}\left(\Delta x^{\prime}, \Delta y^{\prime}\right)=\left|\exp \left(-\frac{\pi^{2} \omega^{2}}{2 \lambda^{2} L_{o}^{2}}\left(x^{\prime 2}+y^{\prime 2}\right)\right)\right|^{2} .
$$

If the theoretical mean speckle sizes $\alpha_{x}$, and $\alpha_{y^{\prime}}$ are defined as the $x^{\prime}$-axis and the $y^{\prime}$-axis values where the normalized autocorrelation function $r_{I}$ of intensity $I$ decreases to $1 /$ e, i.e.

$$
\mid \exp \left(-\left.\frac{\pi^{2} \omega^{2}}{2 \lambda^{2} L_{o}^{2}}\left(\alpha_{x^{\prime}}{ }^{2}+\alpha_{y^{\prime}}{ }^{2}\right)\right|^{2}=\exp (-1),\right.
$$

then

$$
\begin{gathered}
\alpha_{x^{\prime}}=\frac{\lambda L_{o}}{\pi \omega}=\frac{\lambda L_{o}}{\pi \omega_{o} \sqrt{1+\left(\frac{L_{s} \lambda}{\omega_{o}^{2} \pi}\right)^{2}}}, \\
\alpha_{y^{\prime}}=\frac{\lambda L_{o}}{\pi \omega}=\frac{\lambda L_{o}}{\pi \omega_{o} \sqrt{1+\left(\frac{L_{s} \lambda}{\omega_{o}^{2} \pi}\right)^{2}}} .
\end{gathered}
$$

For the next purpose, let us replace (13) and (14) by

$$
\begin{gathered}
\alpha_{x^{\prime}}=\frac{\lambda L_{o}}{\pi \omega_{x^{\prime}}}=\frac{\lambda L_{o}}{\pi \cos \theta_{o} \omega_{o} \sqrt{1+\left(\frac{L_{s} \lambda}{\omega_{o}^{2} \pi}\right)^{2}}}, \\
\alpha_{y^{\prime}}=\frac{\lambda L_{o}}{\pi \omega_{y^{\prime}}}=\frac{\lambda L_{o}}{\pi \omega_{o} \sqrt{1+\left(\frac{L_{s} \lambda}{\omega_{o}^{2} \pi}\right)^{2}}},
\end{gathered}
$$

where $\omega_{x^{\prime}}$ and $\omega_{y^{\prime}}$ represent projections of the Gaussian beam radius $\omega$ into the $x^{\prime}$-axis and the $y^{\prime}$-axis, respectively. The projections are defined by virtue of a nonzero angle $\theta_{o}$ of observation, as is specified in the next section.

\section{RESUlts}

Speckle patterns generated by an object of the size $4 \mathrm{~mm} \times 4 \mathrm{~mm}$ after illumination by the Gaussian beam with the radii $\omega_{o}=40 \mu \mathrm{m}, 50 \mu \mathrm{m}, 60 \mu \mathrm{m}, \quad 70 \mu \mathrm{m}, 80 \mu \mathrm{m}$ and $90 \mu \mathrm{m}$ at its waist situated at the distance $L_{s}=0.2 \mathrm{~m}$ from the object are simulated. The speckle pattern is detected by a matrix detector of the size $13 \mathrm{~mm} \times 13 \mathrm{~mm}$ at the distance $L_{o}=0.4 \mathrm{~m}$ from the object. The object consists of $m_{i} \times n_{i}=400 \times 400$ points and the intensity $I$ of the speckle pattern is detected at $m \times n=300 \times 300$ points. The wavelength of the light is $\lambda=632.8 \mathrm{~nm}$.

In the simulated experimental setup (Fig.1.), the angle between the $x$-axis in the object plane $(x, y)$ and the $x^{\prime}$-axis in the detection plane $\left(x^{\prime}, y^{\prime}\right)$ is $\theta_{o}=30^{\circ}$, whereas the $y$-axis is parallel to the $y^{\prime}$-axis. Hence, the speckle pattern is detected at the nonzero angle of observation $\theta_{o}=30^{\circ}$. Then the projections $\omega_{x^{\prime}}$ and $\omega_{y^{\prime}}$ of the Gaussian beam radius $\omega$ into the $x^{\prime}$-axis and $y^{\prime}$-axis are $\omega_{x^{\prime}}=\omega \cos 30^{\circ}$ and $\omega_{y^{\prime}}=\omega$, respectively.

The achieved numerical results are summarized into the following graphs (Fig.2. - Fig.9.). Firstly, let us explain the results illustrated in Fig.2. and Fig.3. Figs.2. and 3. show the stated mean speckle size as a function of the Gaussian beam radius $\omega_{0}$. The theoretical mean speckle sizes $\alpha_{x^{\prime}}$ and $\alpha_{y^{\prime}}$ (square marks) are computed by means of (15) and (16) after substituting the input parameters of simulation. Values of $\alpha_{x}{ }^{2 \mathrm{D}}, \alpha_{y^{\prime}}{ }^{2 \mathrm{D}}$ and $\alpha_{x^{\prime}}{ }^{1 \mathrm{D}}, \alpha_{y^{\prime}}{ }^{1 \mathrm{D}}$ are stated by means of the method of 2D correlation (circle marks) and the method of 1D correlation (triangle marks), respectively. 


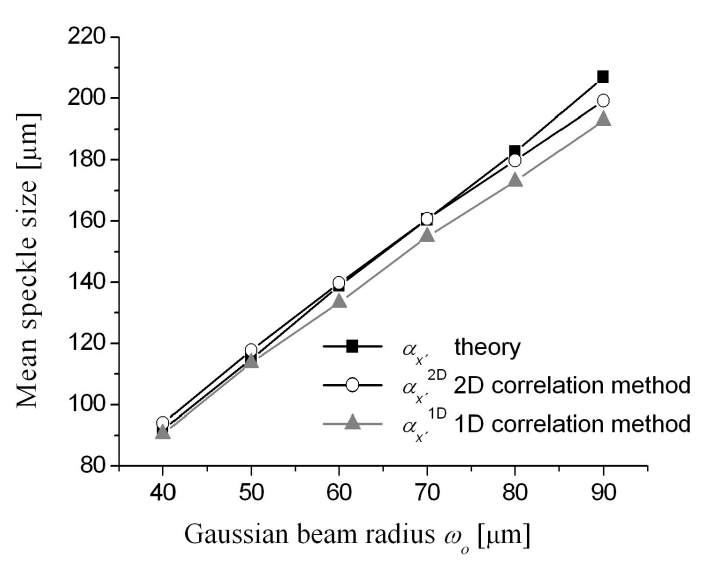

Fig.2. Mean speckle sizes $\alpha_{x^{\prime}}$ (determined from theory), $\alpha_{x^{\prime}}{ }^{2 \mathrm{D}}$ and $\alpha_{x^{\prime}}{ }^{1 \mathrm{D}}$ (stated by the 2D and 1D correlation methods) as a function of the Gaussian beam radius $\omega_{o}$ at its waist. The size of the matrix detector sampled by $300 \times 300$ points is $13 \mathrm{~mm} \times 13 \mathrm{~mm}$.

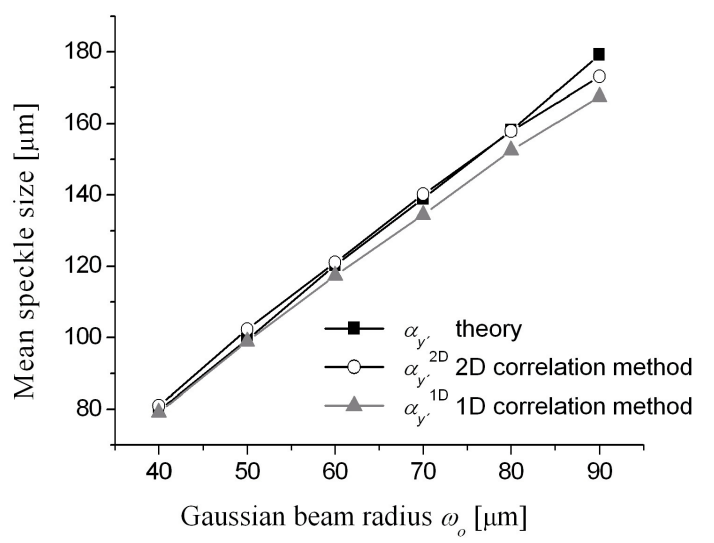

Fig.3. Mean speckle sizes $\alpha_{y^{\prime}}$ (determined from theory), $\alpha_{y^{\prime}}{ }^{2 \mathrm{D}}$ and $\alpha_{y^{\prime}}{ }^{1 \mathrm{D}}$ (stated by the 2D and 1D correlation methods) as a function of the Gaussian beam radius $\omega_{o}$ at its waist. The size of the matrix detector sampled by $300 \times 300$ points is $13 \mathrm{~mm} \times 13 \mathrm{~mm}$.

For illustration of differences among all stated values of $\alpha_{x^{\prime}}{ }^{2 \mathrm{D}}, \alpha_{y^{\prime}}{ }^{2 \mathrm{D}}$ and $\alpha_{x^{\prime}}{ }^{1 \mathrm{D}}, \alpha_{y^{\prime}}{ }^{1 \mathrm{D}}$ and the theoretical mean speckle sizes $\alpha_{x}^{\prime}, \alpha_{y^{\prime}}$, Fig.4. is presented. Fig.4. shows how relative errors $\rho\left(\alpha_{x^{\prime}}{ }^{2 \mathrm{D}}\right), \rho\left(\alpha_{y^{\prime}}{ }^{2 \mathrm{D}}\right)$ and $\rho\left(\alpha_{x^{\prime}}{ }^{1 \mathrm{D}}\right), \rho\left(\alpha_{y^{\prime}}{ }^{1 \mathrm{D}}\right)$ of the stated mean speckle sizes depend on the theoretical mean speckle sizes $\alpha_{x^{\prime}}, \alpha_{y^{\prime}}$ for the size $13 \mathrm{~mm} \times 13 \mathrm{~mm}$ of the matrix detector.

The graphs in Fig.2., Fig.3., and Fig.4. show that the mean speckle sizes $\alpha_{x^{\prime}}{ }^{2 \mathrm{D}}$ and $\alpha_{y^{\prime}}{ }^{2 \mathrm{D}}$ stated by means of the method of $2 \mathrm{D}$ correlation are approximately equal to the theoretical mean speckle sizes $\alpha_{x}$ and $\alpha_{y^{\prime}}$ in the presented range of $\omega_{o}$. Except for the values of $\alpha_{x}{ }^{2}{ }^{2 \mathrm{D}}$ and $\alpha_{y^{\prime}}{ }^{2 \mathrm{D}}$ for $\omega_{o}=90 \mu \mathrm{m}$ (Fig.2. and Fig.3.), the stated relative errors $\rho\left(\alpha_{x}{ }^{2 D}\right)$ and $\rho\left(\alpha_{y^{\prime}}{ }^{2 \mathrm{D}}\right)$ do not exceed $3 \%$ (Fig.4.).

On the other hand, the mean speckle sizes $\alpha_{x^{\prime}}{ }^{1 \mathrm{D}}$ and $\alpha_{y^{\prime}}{ }^{1 \mathrm{D}}$ stated through the method of 1D correlation are for higher theoretical values of the mean speckle sizes $\left(\alpha_{x^{\prime}}, \alpha_{y^{\prime}} \geq 140 \mu \mathrm{m}\right)$ apparently lower than the theoretical mean speckle sizes $\alpha_{x^{\prime}}$ and $\alpha_{y^{\prime}}$. For $\alpha_{x^{\prime}}, \alpha_{y^{\prime}} \geq 140 \mu \mathrm{m}$ the relative errors $\rho\left(\alpha_{x^{\prime}}{ }^{1 \mathrm{D}}\right)$ and $\rho\left(\alpha_{y^{\prime}}{ }^{1 \mathrm{D}}\right)$ are higher than $3 \%$ (Fig.4.). The reason is that a large number of speckles is more necessary for the application of the method of 1D correlation than the method of 2D correlation. The speckle patterns with larger speckles $\left(\alpha_{x^{\prime}}, \alpha_{y^{\prime}} \geq 140 \mu \mathrm{m}\right)$ obviously do not fit the condition well.

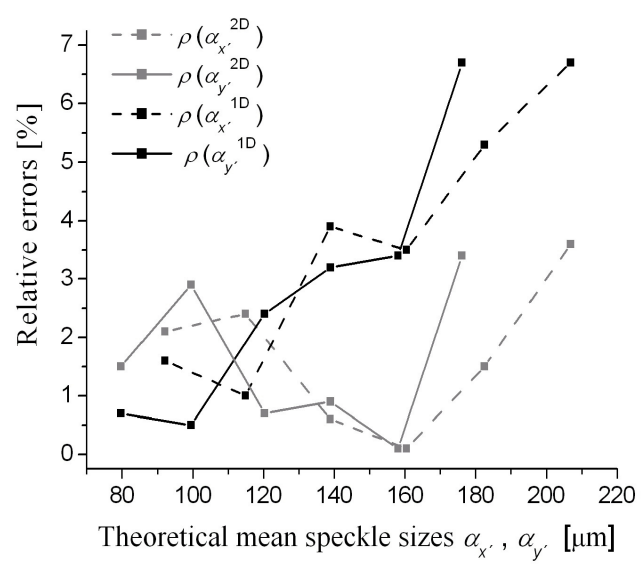

Fig.4. Relative errors $\rho\left(\alpha_{x}{ }^{2 \mathrm{D}}\right), \rho\left(\alpha_{y}{ }^{2 \mathrm{D}}\right), \rho\left(\alpha_{x}{ }^{1 \mathrm{D}}\right)$ and $\rho\left(\alpha_{y}{ }^{1 \mathrm{D}}\right)$ of stated mean speckle sizes determined for the size $13 \mathrm{~mm} \times 13 \mathrm{~mm}$ of the matrix detector.

This fact can be explained as follows. Let us consider one individual speckle of circular shape situated at the matrix detector. Then only the central profile speckle size $\alpha_{x}{ }^{1}{ }^{1} i$ corresponds to real size (radius) of the speckle, whereas the other profile speckle sizes $\alpha_{x}{ }^{1}{ }^{1 D} i$ are evidently smaller than the real speckle size. Subsequently, the resultant mean speckle size $\alpha_{x^{\prime}}{ }^{1 \mathrm{D}}$ computed as an average of all profile speckle sizes $\alpha_{x}{ }^{1 \mathrm{D} i}$ is smaller as well. Nevertheless, as the number of speckles increases, the number of central profile speckle sizes increases, thus resultant mean speckle size $\alpha_{x}{ }^{1 \mathrm{D}}$ corresponds better to the right value.

To testify the above-mentioned consideration, the speckle patterns detected at a larger area of the detector recording a larger number of speckles are simulated and subsequently analyzed. Figs.5. and 6. show the mean speckle size as a function of the Gaussian beam radius $\omega_{o}$ stated from the detector of the size $25 \mathrm{~mm} \times 25 \mathrm{~mm}$. In order to keep the same distance between neighboring points in the matrix detector as in the previous case, the number of points of the detector is $570 \times 570$. The other parameters of the simulation remain the same. Fig.7. shows how the relative errors $\rho\left(\alpha_{x}{ }^{2}{ }^{2 \mathrm{D}}\right), \rho\left(\alpha_{y^{\prime}}{ }^{2 \mathrm{D}}\right)$ and $\rho\left(\alpha_{x^{\prime}}{ }^{1 \mathrm{D}}\right), \rho\left(\alpha_{y^{\prime}}{ }^{1 \mathrm{D}}\right)$ depend on the theoretical mean speckle sizes $\alpha_{x^{\prime}}, \alpha_{y^{\prime}}$ for the size $25 \mathrm{~mm} \times 25 \mathrm{~mm}$ of the matrix detector.

As results from Fig.5., Fig.6., and Fig.7., the stated mean speckle sizes $\alpha_{x}{ }^{1}{ }^{1 \mathrm{D}}$ and $\alpha_{y^{\prime}}{ }^{1 \mathrm{D}}$ are approximately equal to the theoretical mean speckle sizes $\alpha_{x^{\prime}}$ and $\alpha_{y^{\prime}}$, within a larger range of speckle sizes than in the previous case illustrated in Fig.2., Fig.3., and Fig.4. In this case the stated values of $\alpha_{x}{ }^{1 D}$ and $\alpha_{y^{\prime}}{ }^{1 \mathrm{D}}$ significantly differ from the theoretical mean speckle sizes $\alpha_{x^{\prime}}$ and $\alpha_{y^{\prime}}$ for $\alpha_{x^{\prime}}, \alpha_{y^{\prime}} \geq 180 \mu \mathrm{m}$ (the relative errors $\left.\rho\left(\alpha_{x^{\prime}}{ }^{1 \mathrm{D}}\right), \rho\left(\alpha_{y^{\prime}}{ }^{1 \mathrm{D}}\right)>3 \%\right)$. 


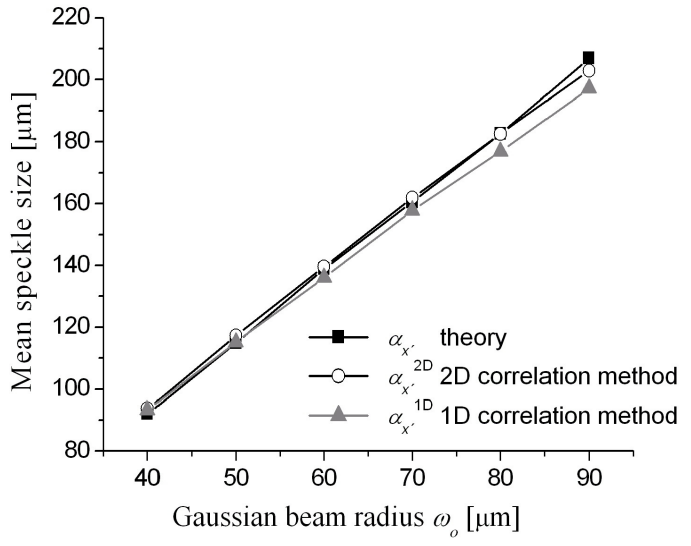

Fig.5. Mean speckle sizes $\alpha_{x^{\prime}}$ (determined from theory), $\alpha_{x}{ }^{2 \mathrm{D}}$ and $\alpha_{x}{ }^{1 \mathrm{D}}$ (stated by the 2D and 1D correlation methods) as a function of the Gaussian beam radius $\omega_{o}$ at its waist. The size of the matrix detector sampled by $570 \times 570$ points is $25 \mathrm{~mm} \times 25 \mathrm{~mm}$.

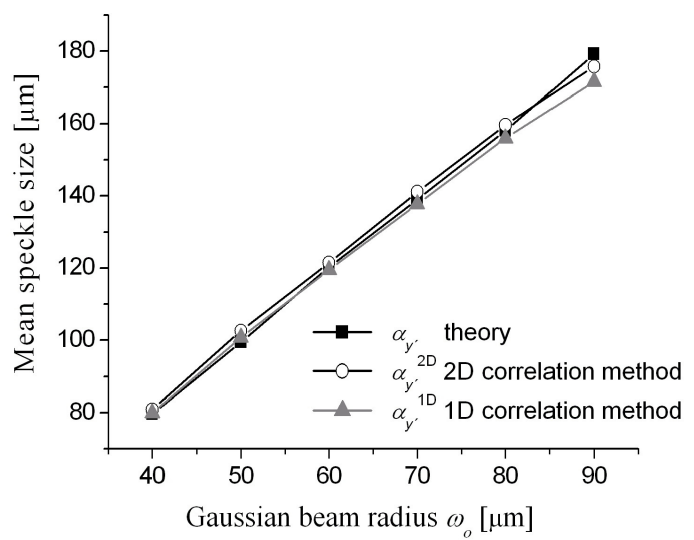

Fig.6. Mean speckle sizes $\alpha_{y^{\prime}}$ (determined from theory), $\alpha_{y^{\prime}}{ }^{2 \mathrm{D}}$ and $\alpha_{y}{ }^{\prime}{ }^{1 D}$ (stated by the 2D and 1D correlation methods) as a function of the Gaussian beam radius $\omega_{o}$ at its waist. The size of the matrix detector sampled by $570 \times 570$ points is $25 \mathrm{~mm} \times 25 \mathrm{~mm}$.

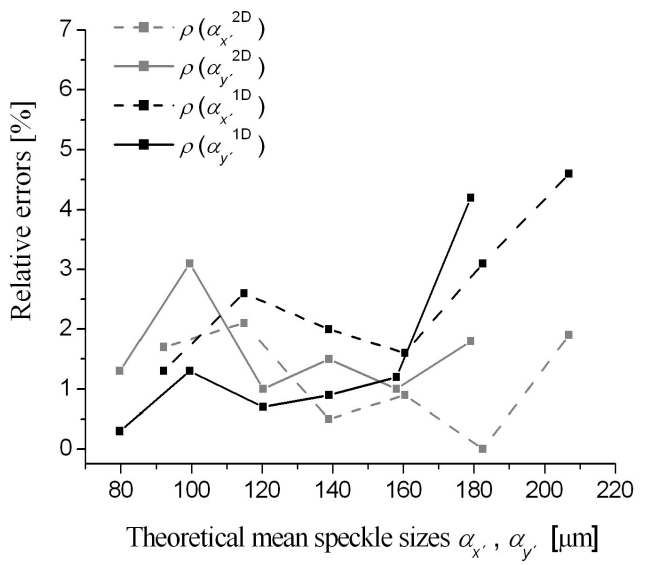

Fig.7. Relative errors $\rho\left(\alpha_{x}{ }^{2 \mathrm{D}}\right), \rho\left(\alpha_{y}{ }^{2 \mathrm{D}}\right), \rho\left(\alpha_{x}{ }^{\prime} \mathrm{D}\right)$ and $\rho\left(\alpha_{y^{\prime}}{ }^{1 \mathrm{D}}\right)$ of stated mean speckle sizes determined for the size $25 \mathrm{~mm} \times 25 \mathrm{~mm}$ of the matrix detector.

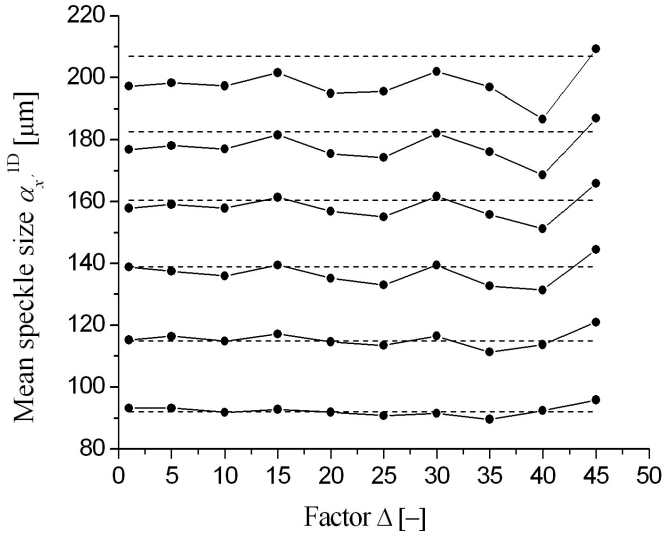

Fig.8. Stated mean speckle size $\alpha_{x^{\prime}}{ }^{1 \mathrm{D}}$ as a function of the factor $\Delta$. The size of the matrix detector sampled by $570 \times 570$ points is $25 \mathrm{~mm} \times 25 \mathrm{~mm}$.

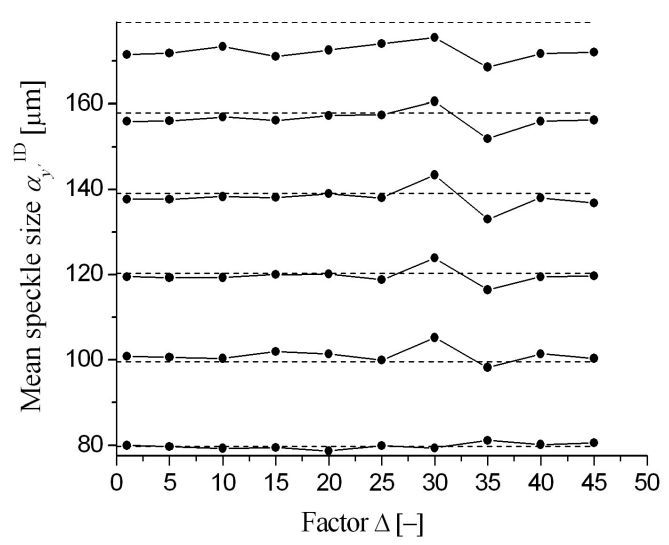

Fig.9. Stated mean speckle size $\alpha_{y^{\prime}}{ }^{1 \mathrm{D}}$ as a function of the factor $\Delta$. The size of the matrix detector sampled by $570 \times 570$ points is $25 \mathrm{~mm} \times 25 \mathrm{~mm}$.

Further, let us focus on results acquired by means of the optimization of the 1D correlation method proposed in section 2. The following graphs (Fig.8. and Fig.9.) show behavior of the mean speckle sizes $\alpha_{x}{ }^{1 \mathrm{D}}$ and $\alpha_{y}{ }^{1 \mathrm{D}}$ computed by (6) and (7) as a function of the factor $\Delta$. The theoretical mean speckle sizes $\alpha_{x^{\prime}}$ and $\alpha_{y^{\prime}}$ are represented by dashed lines. As can be seen, values of $\alpha_{x^{\prime}}{ }^{1 \mathrm{D}}$ and $\alpha_{y^{\prime}}{ }^{1 \mathrm{D}}$ do not change dramatically with the factor $\Delta$ within the interval $\Delta \in[1,20]$. Hence, the minimum number of rows (columns) of the matrix detector $(m \times n=570 \times 570)$, which one can evaluate the mean speckle sizes $\alpha_{x}{ }^{1 \mathrm{D}}\left(\alpha_{y^{\prime}}{ }^{1 \mathrm{D}}\right)$ from, is $m / \Delta=570 / 20 \cong 29(n / \Delta=570 / 20 \cong 29)$. Then good results are achieved from relatively small amount of intensity values selected from the whole matrix detector.

\section{CONCLUSIONS}

In this paper two approaches of computation of the mean speckle size using both the 2D and the 1D normalized autocorrelation function $r_{I}$ of intensity $I$ are applied on the 
simulated speckle patterns. The obtained numerical results are compared with the results obtained by the theoretical relations.

It is shown, that within the presented range of speckle size accuracy of determination of the mean speckle sizes $\alpha_{x}{ }^{2}{ }^{2 \mathrm{D}}, \alpha_{y}{ }^{2 \mathrm{D}}$ acquired by means of the $2 \mathrm{D}$ correlation method does not dramatically depend on the size of the matrix detector. Reasonable results are achieved for both smaller and larger size of the matrix detector $(13 \mathrm{~mm} \times 13 \mathrm{~mm}$ and $25 \mathrm{~mm} \times 25 \mathrm{~mm}$ ).

On the other hand, in the case of the 1D correlation method, more accurate results of determination of the mean speckle sizes $\alpha_{x^{\prime}}{ }^{1 \mathrm{D}}, \alpha_{y^{\prime}}{ }^{1 \mathrm{D}}$ are obtained by using the larger area of the detector $(25 \mathrm{~mm} \times 25 \mathrm{~mm})$. The reason is that the larger number of speckles in the speckle pattern is detected, and therefore, more meaningful evaluation of the speckle size through the 1D correlation method is performed. The range of determination of the mean speckle sizes $\alpha_{x}{ }^{1 \mathrm{D}}, \alpha_{y^{\prime}}{ }^{1 \mathrm{D}}$ by means of the $1 \mathrm{D}$ correlation method increases approximately to $160 \mu \mathrm{m}$, as the area of detector increases to $25 \mathrm{~mm} \times 25 \mathrm{~mm}$ (Fig.5. - Fig.7.).

Further, the optimization of the method of 1D correlation is proposed. The optimization is based on decimation of the $2 \mathrm{D}$ intensity signal representing the detected speckle patterns by the factor $\Delta$. It is shown that to get reasonable results of evaluation of the mean speckle size, one can select only several rows (columns) from the whole matrix detector. Hence, the main advantage of the optimized method of 1D correlation rests on the possibility of determining the mean speckle size from a relatively small amount of detected intensity values, which can positively influence the data processing speed. Moreover, since some types of matrix detectors are able to decimate the 2D signal from the area of the detector, then lower amount of data from the detector can be transferred to the computer, which can additionally improve the effectiveness of the data processing.

\section{ACKNOWLEDGEMENT}

The authors gratefully acknowledge the Grant of the Czech Science Foundation No. 13-12301S and the Grant of the Ministry of Education, Youth and Sports of the Czech Republic No. LG13007.

\section{REFERENCES}

[1] Dainty, J.C. (ed.) (1984). Laser Speckle and Related Phenomena (2nd ed.). Springer-Verlag.

[2] Lehmann, P. (1999). Surface-roughness measurement based on the intensity correlation function of scattered light under speckle-pattern illumination. Applied Optics, 38 (7), 1144-1157.

[3] Chicea, D. (2007). An alternative algorithm to calculate the biospeckle size in coherent light scattering experiments. Romanian Journal of Physics, 54 (1-2), 147-155.

[4] Piederriere, Y., Meur, J.L., Cariou, J., Abgrall, J.F., Blouch, M.T. (2004). Particle aggregation monitoring by speckle size measurement; application to blood platelets aggregation. Optics Express, 12 (19), 45964601.

[5] Piederriere, Y., Cariou, J., Guern, Y., Jeune, B.L., Brun, G.L., Lotrian, J. (2004). Scattering through fluids: Speckle size measurement and Monte Carlo simulation close to and into the multiply scattering. Optics Express, 12 (1), 176-188.

[6] Hamarová, I., Šmíd, P., Horváth, P., Hrabovský, M. (2014). A simulation analysis of an extension of onedimensional speckle correlation method for detection of general in-plane translation. The Scientific World Journal, article ID 704368.

[7] Hamarová, I., Horváth, P., Šmíd, P., Hrabovský, M. (2012). The simulation of the origin and propagation of the speckle field generated through a plane wave and Gaussian beam and its verification by speckle correlation method. Optik, 123 (5), 404-408.

[8] Hamarová, I., Horváth, P., Šmíd, P., Hrabovský, M. (2010). Computer simulation of the speckle field propagation. In Proceedings of SPIE 7746. SPIE, 77460M-1-77460M-8.

[9] Gonzales, R.C., Woods, R.E. (2008). Digital Image Processing (3th ed.). Pearson Education Inc.

[10] Saleh, B.E.A., Teich, M.C. (1991). Fundamentals of Photonics. John Wiley \& Sons.

Received August 9, 2013. Accepted June 10, 2014. 